\title{
Wo kann Suizidprävention ansetzen? Vorschlag eines 6-Phasen-Modells suizidaler Krisen
}

\author{
Where can Suicide Prevention be Applied? Proposal of a 6-Phase Model \\ for Suicidal Crises
}

Autor

Institut
Thomas Reisch

Universitätsklinik und Poliklinik für Psychiatrie Bern, Schweiz

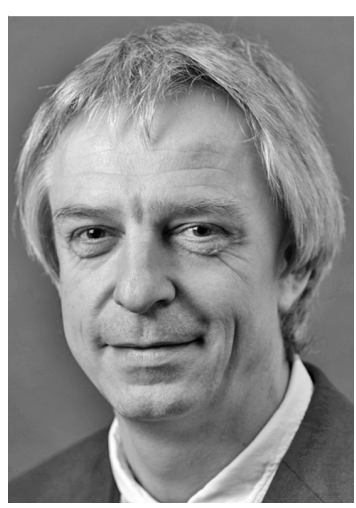

PD Dr. med. Thomas Reisch

\section{Bibliografie \\ Dol http://dx.doi.org/ 10.1055/s-0032-1305205 Psychiat Prax 2012; 39: 257-258 \\ (c) Georg Thieme Verlag KG Stuttgart · New York ISSN 0303-4259}

\section{Korrespondenzadresse}

PD Dr. med. Thomas Reisch Ärztlicher Leiter Schwerpunkt Psychotherapie, Universitätsklinik und Poliklinik für Psychiatrie

Bolligenstraße 111 3000 Bern 60, Schweiz thomas.reisch@gef.be.ch
Suizid ist die häufigste direkte Todesursache in der Psychiatrie [1, 2]. Im Gegensatz zu anderen Todesursachen finden sich relativ viele jüngere Menschen, die durch Suizid versterben, woraus sich eine hohe Anzahl verlorener Lebensjahre [3] ergibt, in der Schweiz fallen etwa $15 \%$ aller verlorenen Lebensjahre auf den Suizid. Schon deshalb sollte es ein Bestreben der Politik und Psychiatrie sein, diese Zahl zu verringern. Die Evidenz für die Suizidprävention ist insgesamt hoch, für verschiedene Public-Health-Maßnahmen und einige vorwiegend psychotherapeutische Maßnahmen konnte deren Wirksamkeit wiederholt nachgewiesen werden [4].

Mehrere Autoren haben Modelle zur Suizidalität entwickelt, welche zumeist die Ursachen eines Suizids zu erklären versuchen. In dem umfassenden Modell von Mann [5] werden verschiedenste prädisponierende Faktoren wie z.B. Genetik, Lebenserfahrungen, Erkrankungen, Substanzmissbrauch, Nahrung und aktuelle Auslöser wie psychiatrische/medizinische Erkrankungen, Stress/ Krisensituationen mit Störungen zentraler Neurotransmitter, insbesondere Serotonin, in Beziehung gesetzt [6]. Diese Modelle sind bedingt durch ihre Komplexität in der Praxis der Suizidprävention häufig schwer vermittelbar. Suizid ist keine Krankheit, sondern in allererster Linie eine Handlung. In der Vermittlung des Ablaufs eines Suizidversuches arbeiten wir daher mit einem verhaltens- und ablauforientierten 6-Phasen-Modell, welches für Patienten und Angehörige häufig eingängiger ist [7]. Natürlich sind die Handlungsabläufe von Menschen, welche sich suizidieren, hoch individuell und die einzelnen Schritte, welche unser Modell postuliert, werden unterschiedlich durchlaufen und teilweise übersprungen. Dieses Modell und die Implikationen für die Suizidprävention sollen hier skizziert werden (৫ Abb. 1).

1. Präsuizidale Phase: Wir gehen davon aus, dass die meisten Menschen auf der Basis einer klini- schen oder subklinischen Störung, am häufigsten der depressiven Symptomatik, eine zwischenzeitlich erhöhte Vulnerabilität haben. Suizidprävention in dieser vorsuizidalen Phase liegt in der psychiatrisch-psychotherapeutischen Behandlung der psychiatrischen Grundleiden.

2. Mental-Pain-Phase: Prototypisch kommt es unter diesen Voraussetzungen durch Stressoren zu einer subjektiv unerträglichen Situation. Zumeist ist dies bedingt durch einen gemischt externer und interner Stimulus (z.B. das Ende einer Partnerschaft und der Gedanke, dass die dadurch ausgelöste emotionale Krise unerträglich ist). Es kommt zu einem äußerst aversiven Emotionszustand, welchen Shneidman [8] als Psychache und Orbach et al. [9] als Mental Pain bezeichnete. In dieser ersten Phase werden die Menschen von der Außenwelt zwar nicht automatisch als suizidal, aber zumindest als leidend wahrgenommen. In der subjektiven Notsituation sind Zugriffe auf vergangene positive ebenso wie mögliche antizipierte positive Erlebnisse nicht möglich. Auch die Verantwortung für das direkte soziale Umfeld (Was tue ich anderen durch einen Suizid an?) wird nicht mehr wahrgenommen. Es gibt nur noch den einen Fokus: Wie kann ich dieses Leiden beenden? Das rationale Denken ist nur erschwert möglich bei deutlich geänderter Hirnaktivität [10], demzufolge greifen Betroffene auf bekannte oder subjektiv naheliegende Lösungen zurück wie z.B. Zeitungsberichte (Werther-Effekt) oder Suizide von Nahestehenden oder eigene frühere suizidale Handlungen, welche im Vorfeld des Suizids gedanklich oder auf der Handlungsebene mehrfach durchlaufen werden. Aus diesem Grunde ist eine Verhinderung des Werther-Effektes durch Medienguidelines wichtig, daher benutzen Brückenspringer Brücken, über die sie auch im Alltag gehen, und darum benutzen Menschen nach früherer Selbstintoxikation wieder die gleichen Medikamente. Die Reduktion der Verfügbarkeit von Suizidmethoden greift hier an. 


\begin{tabular}{|c|c|c|}
\hline Phasen & & Suizidpräventionsmaßnahme \\
\hline 1. Präsuizidale Phase & $\longleftarrow$ & $\begin{array}{c}\text { Behandlung der psychiatrischen Erkrankung } \\
\text { Weiterbildung Hausärzte }\end{array}$ \\
\hline 2. Mental-Pain-Phase & $\longleftarrow$ & $\begin{array}{l}\text { Weiterbildung Hausärzte } \\
\text { Psychiatrische Krisenintervention } \\
\text { (Schulung der Medienschaffenden) } \\
\text { (Methodenrestriktion) }\end{array}$ \\
\hline $\begin{array}{l}\text { 3. Suizidhandlungs- } \\
\text { phase }\end{array}$ & & Schulung Ärzte und Pflegepersonal \\
\hline $\begin{array}{c}\text { 4. Finale } \\
\text { Ambivalenzphase }\end{array}$ & & $\begin{array}{c}\text { Schulung Polizei, Taxifahrer etc. } \\
\text { Maßnahmen zur Verbesserung } \\
\text { der Zivilcourage }\end{array}$ \\
\hline $\begin{array}{l}\text { 5. Finale } \\
\text { Handlungsphase }\end{array}$ & $\leftarrow$ & $\begin{array}{l}\text { Schulung Polizei, Taxifahrer etc. } \\
\text { (effizientes Notfallsystem) }\end{array}$ \\
\hline 6. Aufwachen & $\longleftarrow$ & $\begin{array}{l}\text { Nachbetreuung von Patienten nach } \\
\text { Suizidversuch }\end{array}$ \\
\hline
\end{tabular}

Abb. 1 6-Phasen-Modell des Suizids.

3. Erste Suizidhandlungsphase: Ist die vermeintliche Lösungsmöglichkeit zur Aufhebung des mentalen Schmerzes gefunden, setzt die erste Suizidhandlungsphase ein. Dadurch, dass das Problem subjektiv gelöst wurde, ist der Schmerz nicht mehr im Vordergrund. Der suizidale Mensch wird hier als relativ entspannt wahrgenommen und die Gefahr des Suizids wird verkannt. Klinisch handelt es sich hier um ein dissoziationsähnliches Zustandsbild, in dem der mentale Schmerz abgespalten wird und die Person in einer Art „Autopilot“ Vorbereitungshandlungen durchführt. Psychiatriefachkräfte erleben Patienten, die sich später suizidierten, bei dem letzten Kontakt (z. B. Entlassung in den Urlaub) häufig als ruhig und entspannt. Ein schneller Wechsel von Anspannung auf Entspannung sollte daher im klinischen Setting als Alarmzeichen nicht verpasst werden. Bei genauem Hinsehen gibt es aber sehr wohl Unterschiede zu einer normalen Symptombesserung. Beschrieben werden häufig eine Wortarmut, der fehlende oder geringe Blickkontakt und die fehlende konkrete Planung der nahen Zukunft der Betroffenen.

4. Finale Ambivalenzphase: Von Aufzeichnungen in Bahnhöfen und Brücken wissen wir, dass viele Menschen unmittelbar vor der letzten Handlung noch einmal innehalten und die angefangene Handlung unterbrechen: Sie lassen Züge durchfahren oder warten am Brückengeländer. Videoaufzeichnungen von Patienten, welche kurze Zeit später von Brücken oder im Bahnhof vor den Zug sprangen, zeigen hier ein nahezu uniformes Bild: Die Betroffenen stehen mit verschränkten Armen nach vorne gebeugt und wirken äußerst gespannt, der Blick ist nach unten gerichtet. An dieser Stelle gelingt es, Passanten oder Professionellen immer wieder, die Suizidalen zur Umkehr zu bewegen. Dieses direkte Verhindern der suizidalen Handlung ist wahrscheinlich sogar die effektivste Suizidprävention. Nur etwa $5 \%$ derjenigen, welche vom Sprung von der Golden Gate Bridge abgehalten wurden, suizidierten sich in den nächsten 26 Jahren [11]. Dies impliziert aber auch mehrere Formen der Suizidprävention: Berufsfachkräfte (Polizisten, Taxifahrer, Zugführer) können geschult werden, Suizidale zu erkennen und die nötigen Schritte einzuleiten. Eine Förderung der Zivilcourage der Bevölkerung wäre hier eine sinnvolle Maßnahme.

5. Finale Handlungsphase: Bei einigen Suizidmethoden wie z.B. dem Brückensprung sind die finale Ambivalenzphase und die fi- nale Handlungsphase, also das Ausführen der potenziell zum Tode führenden Handlung, überlappend. Selten ist es möglich, die eigentliche Suizidhandlung zu unterbrechen. Die oben zitierte Studie von Seiden [11] zeigt dies deutlich. Einige der geretteten Menschen waren bereits jenseits des Geländers der Brücke und wurden aktiv vom Sprung abgehalten.

6. Aufwachen: Die allermeisten Menschen, welche einen Suizidversuch überleben, bereuen ihre Suizidhandlung, einige hiervon bereits kurz nach Einleitung der Tat. Von Brückenspringern wissen wir, dass dieses „Aufwachen“, das Ende des dissoziativen Zustands, bereits während des Falls einsetzen kann. Bei vielen Suizidmethoden ist die begonnene Handlung nicht mehr umkehrbar (Erhängen, Erschießen, Zug und Sprung), das Aufwachen kommt hier zu spät. Eine Suizidprävention durch Restriktion der Suizidmethoden hat daher hier eine hohe Priorität bei hoch letalen und nicht mehr umkehrbaren Handlungen. Eine weitere Maßnahme kurz nach dieser Phase ist die Nachbehandlung von Patienten nach einem Suizidversuch. Das therapeutische Fenster ist hier allerdings oft nur sehr kurz. Einige Patienten gehen oft erstaunlich schnell wieder zur Tagesordnung über und viele sind nicht bereit, ihre Suizidhandlungsmuster anzusehen, um daraus Bewältigungshilfen für zukünftige Krisen abzuleiten. Wünschenswert ist es daher aus der Sicht der Suizidprävention, eine Versorgungsstruktur anzubieten, welche ermöglicht, dass ein Patient nach einem Suizidversuch relativ zeitnah im ambulanten Rahmen durch die gleiche Person wieder gesehen wird. In der Realität findet hier aber zumeist, bedingt durch die Struktur der Institution, ein Wechsel der Betreuungsperson statt.

Suizidprävention kostet Geld. Zumindest in der Schweiz werden bislang aber von staatlicher Seite wenige Gelder in die Suizidprävention investiert. Insofern stellt sich die Frage nicht, ob wir zu viel in die Suizidprävention stecken. Viele der vorgestellten Suizidpräventionsmaßnahmen kosten verhältnismäßig wenig in Anbetracht des Gewinns vieler Menschenleben. Insofern gilt: Es gibt noch viel zu tun!

\section{Literatur}

1 Neuner T, Hübner-Liebermann B, Hausner H et al. Kleine Zahlen, große Ergebnisse: „Suizidhochburg“ Weiden? Psychiat Prax 2011; 38: 253 255

2 Neuner T, Mehlsteibl D, Hübner-Liebermann B et al. Risikoprofile für den Kliniksuizid schizophrener und depressiver Patienten - eine psychologische Autopsiestudie. Psychiat Prax 2010; 37: 119-126

3 OBSAN. Indikator Verlorene Potentielle Lebensjahre. 2004: http:// www.obsandaten.ch/indikatoren/2_3_1/2004/d/231.pdf

4 Mann JJ, Apter A, Bertolote J et al. Suicide prevention strategies: a systematic review. JAMA 2005; 294: 2064-2074

5 Mann JJ, Waternaux C, Haas GL et al. Toward a clinical model of suicidal behavior in psychiatric patients. Am J Psychiatry 1999; 156: 181-189

6 Lyddon R, Dwork AJ, Keddache $M$ et al. Serotonin 2c receptor RNA editing in major depression and suicide. World J Biol Psychiatry 2001: March 9 [Epub ahead of print]

7 Michel K, Valach L. Suicide as goal-directed action. Archives of Suicide Research 1997; 3: 213-221

8 Shneidman ES. Suicide As Psychache: A Clinical Approach To Self-Destructive Behavior. Northvale, NJ/London: Jason Aronson, Inc; 1993: 258

9 Orbach I, Mikulincer M, Gilboa-Schechtman E et al. Mental Pain and Its Relationship to Suicidality and Life Meaning. Suicide and Life-Threatening Behavior 2003; 33: 231 - 241

10 Reisch T, Seifritz E, Esposito F et al. An fMRI Study on Mental Pain and Suicidal Behavior. Journal of Affective Disorder 2010; 126: 321 - 325

11 Seiden $R H$. Where are they now? A follow-up study of suicide attempters from the Golden Gate bridge. Suicide Life Threat Behav 1978; 8: $203-216$ 